\title{
Study of Hilbert algebras in point of filters
}

\author{
A. Soleimani Nasab and A. Borumand Saeid
}

\begin{abstract}
The aim of this work is to introduce some types of filters in Hilbert algebras. Some theorems are stated and proved which determine the relationship between these notions and other filters of Hilbert algebra and by some examples we show that these concepts are different. The relationships between these filters and quotient algebras that are constructed via these filters are described.
\end{abstract}

\section{Introduction}

The variety of Hilbert algebras is an important tool for investigations in intuitionistic logic and other non-classical logics. Hilbert algebras represent the algebraic counterpart of the implicative fragment of Intuitionistic Propositional Logic. Hilbert algebra was studied by A. Diego[8] and D. Buşneag [1, 2, 3, 4], S. A. Celani, D. Montangie [7], S. A. Celani [5], S. M. Hong, Y. B. Jun[11] and Figallo et al.[9].

The aim of this work is to study filter theory in Hilbert algebras. The concepts positive implicative filters, Boolean filters and fantastic filters in Hilbert algebras are introduced and relationships between these filters and quotient algebras that are constructed via these filters are described.

The structure of the paper is as follows: in Section 2, some definitions and properties about Hilbert algebras are recalled. In Section 3, implicative filters are developed in Hilbert algebras. In Section 4, the notion positive implicative filter in Hilbert algebras is introduced, and we characterized them. In

Key Words: Hilbert algebra (with infimum, with supremum), (implicative, positive implicative, Boolean, fantastic) filter, Boolean algebra.

2010 Mathematics Subject Classification: 03B47, 03G25, 06D99.

Received: 07.01.2015

Accepted: 02.03.2015 
Section 5, the notion fantastic filter is defined in Hilbert algebra. We give relationships between fantastic filters and other filters. In Section 6, the notion Boolean filters in Hilbert algebras is developed and we prove that the positive implicative filters, fantastic filters and Boolean filters in bounded Hilbert algebras are equivalent. In Section 7, prime filters of the first kind, prime filters of the second kind and prime filters of the third kind are introduced and prime filters in Hilbert algebras are characterized.

\section{Preliminaries}

We recall some basic definitions and results that are necessary for this paper.

A Hilbert algebra is an algebra $(H, \rightarrow, 1)$ of type $(2,0)$ such that the following axioms hold, for all $x, y, z \in H$ :

$(H 1) x \rightarrow(y \rightarrow x)=1$;

$(H 2)(x \rightarrow(y \rightarrow z)) \rightarrow((x \rightarrow y) \rightarrow(x \rightarrow z))=1 ;$

(H3) if $x \rightarrow y=y \rightarrow x=1$, then $x=y$.

It is proved that the above definition is equivalent to the system $\left\{H_{4}, H_{5}, H_{6}, H_{7}\right\}$, where:

(H4) $x \rightarrow x=1$;

(H5) $1 \rightarrow x=x$

$(H 6) x \rightarrow(y \rightarrow z)=(x \rightarrow y) \rightarrow(x \rightarrow z) ;$

$(H 7)(x \rightarrow y) \rightarrow((y \rightarrow x) \rightarrow x)=(y \rightarrow x) \rightarrow((x \rightarrow y) \rightarrow y)$.

For a Hilbert algebra $H,(H, \leq)$ is a poset by defining an order relation $\leq$ as $x \leq y$ if and only if $x \rightarrow y=1$. (called the natural order on $H$ ), with respect to this order, 1 is the greatest element of $H$. If $H$ has a smallest element 0 , we say that $H$ is bounded, in this case we define a unary operation $*$ as $x^{*}=x \rightarrow 0$, for each $x \in H$.

A Hilbert algebra is prelinear if $(x \rightarrow y) \vee(y \rightarrow x)=1$, for all $x, y \in H$. Linear (i.e., totally ordered) Hilbert algebras are prelinear. In linear Hilbert algebra with supremum, 1 is $\vee$-irreducible. This means that for all $x$ and $y \in H, x \vee y=1$ if and only if $x=1$ or $y=1$.

Example 2.1. [1] 
(i) If $(H, \leq)$ is a poset with 1 , then $(H, \rightarrow, 1)$ is a Hilbert algebra, where $x, y \in H$,

$$
x \rightarrow y= \begin{cases}1 & \text { if } x \leq y \\ y & \text { if } x \not \leq y\end{cases}
$$

(ii) If $(H, \wedge, \vee, \rightarrow, 0)$ is a Heyting algebra, then $(H, \rightarrow, 1)$ is a Hilbert algebra.

(iii) There are Hilbert algebras which are not Heyting or Boolean algebras [8].

The following proposition include some properties of Hilbert algebra $[1,4$, 8].

Proposition 2.2. In each Hilbert algebra $H$, the following relations hold for all $x, y, z \in H$,

(h1) $1 \rightarrow x=x, x \rightarrow x=1, x \rightarrow 1=1$;

(h2) $x \leq y \rightarrow x, x \leq(x \rightarrow y) \rightarrow y$;

(h3) $x \rightarrow(x \rightarrow y)=x \rightarrow y$;

(h4) $((x \rightarrow y) \rightarrow y) \rightarrow y=x \rightarrow y$;

(h5) $x \rightarrow(y \rightarrow z)=y \rightarrow(x \rightarrow z)$;

(h6) $x \rightarrow y \leq(y \rightarrow z) \rightarrow(x \rightarrow z)$;

(h7) $x \rightarrow y \leq(z \rightarrow x) \rightarrow(z \rightarrow y)$;

(h8) If $x \leq y$, then $y \rightarrow z \leq x \rightarrow z$ and $z \rightarrow x \leq z \rightarrow y$;

(h9) $x \rightarrow(y \rightarrow z)=(x \rightarrow y) \rightarrow(x \rightarrow z)$;

(h10) $(x \rightarrow y) \rightarrow((y \rightarrow x) \rightarrow x)=(y \rightarrow x) \rightarrow((x \rightarrow y) \rightarrow y)$;

(h11) $(x \rightarrow y) \rightarrow(y \rightarrow x)=y \rightarrow x$;

(h12) $((x \rightarrow y) \rightarrow x) \rightarrow y=x \rightarrow y$.

Proposition 2.3. [4] If $H$ is a bounded Hilbert algebra and $x, y \in H$, then

(bh1) $0^{*}=1,1^{*}=0$;

(bh2) $x \rightarrow y^{*}=y \rightarrow x^{*}$; 
(bh3) $x \rightarrow x^{*}=x^{*}, x^{*} \rightarrow x=x^{* *}, x \leq x^{* *}, x \leq x^{*} \rightarrow y$;

(bh4) $x \rightarrow y \leq y^{*} \rightarrow x^{*}$;

(bh5) If $x \leq y$, then $y^{*} \leq x^{*}$;

(bh6) $x^{* * *}=x^{*}$;

(bh7) $(x \rightarrow y)^{* *}=x \rightarrow y^{* *}=x^{* *} \rightarrow y^{* *}$;

Definition 2.4. [9] A Hilbert algebra $(H, \rightarrow, 1)$ is called a Hilbert algebra with infimum when the underlying structure $(H, \leq)$ with the order induced by $\rightarrow$ is a meet- semilattice.

Or, equivalently

Definition 2.5. [9] A Hilbert algebra with infimum is an algebra $(H, \rightarrow, \wedge, 1)$ of type $(2,2,0)$ which satisfies the following conditions:

(i) The reduct $(H, \rightarrow, 1)$ is a Hilbert algebra.

(ii) These identities are verified:

(1) $x \wedge(y \wedge z)=(x \wedge y) \wedge z$

(2) $x \wedge x=x$;

(3) $x \wedge(x \rightarrow y)=x \wedge y$;

(4) $(x \rightarrow(y \wedge z)) \rightarrow((x \rightarrow z) \wedge(x \rightarrow y))=1$.

Example 2.6. Let $(H, \wedge, 1)$ be a meet- semilattice with element 1 . Then $(H, \wedge, \rightarrow, 1)$ which

$$
x \rightarrow y= \begin{cases}1 & \text { if } x \leq y \\ y & \text { otherwise }\end{cases}
$$

is a Hilbert algebra with infimum.

It is proved that the order determined by the structure of the meetsemilattice coincides with the order determined by the structure of a Hilbert algebra [9]. Thus $x \leq y$ if and only if $x=x \wedge y$ if and only if $x \rightarrow y=1$.

We say that a Hilbert algebra with infimum has the property $(P)$ if for every $x, y \in H$,

$$
(P): x \rightarrow(y \rightarrow(x \wedge y))=1
$$

Theorem 2.7. [4] Let $H$ be a Hilbert algebra with infimum. The following assertions are equivalent: 
(i) $H$ has the property $(P)$;

(ii) For every $x, y, z \in H, x \rightarrow(y \rightarrow z)=(x \wedge y) \rightarrow z$;

(iii) For every $x, y, z \in H, x \wedge(y \rightarrow z)=x \wedge[(x \wedge y) \rightarrow(x \wedge z)]$.

We note that the Hilbert algebras with infimum satisfying the property $(P)$ are Brouwerian semilattices [12], also called Implicative semilattices [14], or also called Hertz algebras [16].

We recall that an Implicative semilattice [6] is an algebra $(A, \wedge, \rightarrow, 1)$ in which $(A, \wedge)$ is a semilattice and for all $x, y, z \in A$,

$$
z \leq x \rightarrow y \text { if and only if } z \wedge x \leq y .
$$

If $H$ is a Hilbert algebra with infimum satisfying the property $(P)$, then

$$
x \rightarrow(y \rightarrow z)=(x \wedge y) \rightarrow z .
$$

So, $x \rightarrow(y \rightarrow z)=1$ iff $(x \wedge y) \rightarrow z=1$, and as $a \leq b$ iff $a \rightarrow b=1$, for all $a, b \in H$, we get that $x \leq y \rightarrow z$ iff $x \rightarrow(y \rightarrow z)=1$ iff $(x \wedge y) \rightarrow z=1$ iff $(x \wedge y) \leq z$. Thus, $H$ is an implicative semilattices.

Definition 2.8. [7] An algebra $(H, \rightarrow, \vee, 1)$ of type $(2,2,0)$ is a Hilbert algebra with supremum or sH-Hilbert algebra if

(i) $(H, \rightarrow, 1)$ is a Hilbert algebra;

(ii) $(H, \vee, 1)$ is a join-semilattice with last element 1.

(iii) For all $a, b \in H, a \rightarrow b=1$ if and only if $a \vee b=b$.

Example 2.9. [7]

(i) In every join-semilattice $(H, \vee, 1)$ with last element 1 it is possible to define a structure of Hilbert algebra with supremum considering the implication $\rightarrow$ defined by the order, i.e., $a \rightarrow b=b$ if $a \nless b$, and $a \rightarrow b=1$ if $a \leq b$.

(ii) The Boolean lattice with two atoms $B_{2}=\{0, a, b, 1\}$ with the implication $\rightarrow$ defined by the order is a Hilbert algebra where the supremum exists for any pair of elements but it is not a Heyting algebra.(An atom of a Boolean algebra is an element $x$ such that there exist exactly two elements $y$ satisfying $y \leq x$, namely $x$ and 0 .) 
Remark 2.10. [2] If $H$ is a Hilbert algebra without 0 , then by adding a new element $0 \notin H$ and define in $H^{\prime}=H \cup\{0\}$ the implication as in table:

\begin{tabular}{c|ccc}
$\rightarrow$ & 0 & $x$ & 1 \\
\hline 0 & 1 & 1 & 1 \\
$x$ & 0 & 1 & 1 \\
1 & 0 & $x$ & 1
\end{tabular}

(where $x \in H$ ), then $\left(H^{\prime}, \rightarrow, 0,1\right)$ becomes a bounded Hilbert algebra.

Definition 2.11. $[1,2,8]$ A subset $D$ of Hilbert algebra $H$ is called a deductive system (or implication filter or simply filter) of $H$ if

(1) $1 \in D$,

(2) If $x \in D$ and $x \rightarrow y \in D$, then $y \in D$,

for all $x, y \in H$.

We note that, in some papers the deductive system is called implicative filter too. We use implicative filter in this paper.

Theorem 2.12. [8] Let $D$ be a deductive system of Hilbert algebra. If $x \leq y$ and $x \in D$, then $y \in D$.

We denote $D s(H)=\{D: D$ is a deductive system of $H\}$. If $H$ is bounded, then a deductive system $D$ is proper if and only if $0 \notin D$. From now $(H, \rightarrow$ $, 0,1)$ or simply $H$ is a Hilbert algebra.

Theorem 2.13. [11] Let $D$ be a nonempty subset of $H$. Then $D$ is an implicative filter of $H$ if and only if $x \leq y \rightarrow z$ implies $z \in D$, for $x, y \in D$.

Let $F$ be an implicative filter of a bounded Hilbert algebra $H$. Define: $x \equiv_{F} y$ if and only if $x \rightarrow y \in F$ and $y \rightarrow x \in F$. Then $\equiv_{F}$ is a congruence relation on $H$. The set of all congruence classes is denoted by $H / F$, i.e, $H / F:=\{[x]: x \in H\}$, where $[x]=\left\{y \in H \mid x \equiv_{F} y\right\}$. Define $\rightarrow$ on $H / F$ as follows: $[x] \rightarrow[y]=[x \rightarrow y]$, and $1=1 / F=F$.

Therefore $(H / F, \rightarrow,[1],[0])$ is a bounded Hilbert algebra with respect to $F$ and the order relation on $H / F$ is given by $[x] \leq[y]$ if and only if $x \rightarrow y \in F$. Clearly, $[x]=[1]$ if and only if $x \in F$.

Theorem 2.14. [4] For a bounded Hilbert algebra $H$, the following assertions are equivalent: 
(i) $x^{* *}=x$, for every $x \in H$;

(ii) $H$ is a Boolean algebra relative to natural ordering, where $x \wedge y=(x \rightarrow$ $\left.y^{*}\right)^{*}, x \vee y=x^{*} \rightarrow y$.

Corollary 2.15. Let $(H, \rightarrow, 0,1)$ be a bounded Hilbert algebra, which $x^{* *}=x$, for every $x \in H$. Then

(i) $(H, \vee, \wedge, \rightarrow, 0,1)$ is a Heyting algebra;

(ii) $(H, \vee, \wedge, \rightarrow, *, 0,1)$ is a Boolean algebra.

Corollary 2.16. [4] A bounded Hilbert algebra $H$ is a Boolean algebra (relative to natural ordering) if and only if for every $x, y \in H$ we have $(x \rightarrow y) \rightarrow x=x$.

Corollary 2.17. [4] For a bounded Hilbert algebra $H$, the following assertions are equivalent:

(i) $H$ is Boolean algebra (relative to natural ordering);

(ii) $(x \rightarrow y) \rightarrow y=(y \rightarrow x) \rightarrow x$;

(iii) $x^{*} \rightarrow y=y^{*} \rightarrow x$;

(iv) $(x \rightarrow y) \rightarrow y=x \vee y$;

$(v) x^{*} \rightarrow y=x \vee y$.

Definition 2.18. [1] An implicative filter $M$ of a Hilbert algebra $H$ is called maximal if it is not properly contained in any other proper implicative filter of $H$.

Theorem 2.19. [1] An implicative filter $M$ of a bounded Hilbert algebra $H$ is a maximal if and only if for all $x \in H$, if $x \notin M$ then $x^{*} \in M$.

\section{Implicative filters in Hilbert algebras}

In this section we give simple characterizations of implicative filters defined in the previous section.

Theorem 3.1. Let $D \subseteq H, 1 \in D$. Then the following conditions are equivalent, for every $x, y, z \in H$ :

(i) D is an implicative filter;

(ii) If $x \rightarrow(y \rightarrow z) \in D, x \rightarrow y \in D$, then $x \rightarrow z \in D$; 
(iii) If $x \rightarrow y, y \rightarrow z \in D$, then $x \rightarrow z \in D$;

(iv) $H_{a}=\{x \in H: a \rightarrow x \in D\}$ is an implicative filter, for any $a \in H$.

Proof. $(i) \Rightarrow($ ii $)$ Let $x \rightarrow(y \rightarrow z) \in D, x \rightarrow y \in D$. We have $[x \rightarrow(y \rightarrow$ $z)] \leq(x \rightarrow y) \rightarrow(x \rightarrow z)$ thus $(x \rightarrow y) \rightarrow(x \rightarrow z) \in D$, hence $x \rightarrow z \in D$.

$($ ii $) \Rightarrow($ iii $)$. Let $x \rightarrow y, y \rightarrow z \in D$. Then $y \rightarrow z \leq(x \rightarrow y) \rightarrow(x \rightarrow z)$, by Proposition 2.2. Thus $x \rightarrow(y \rightarrow z)=(x \rightarrow y) \rightarrow(x \rightarrow z) \in D$. Since $x \rightarrow y \in D$, hence $x \rightarrow z \in D$ by $(i i)$.

$($ iii $) \Rightarrow(i)$ Let $x, x \rightarrow y \in D$. Since $x=1 \rightarrow x, x \rightarrow y \in D$ thus $y=1 \rightarrow y \in D$ by $($ iii $)$. Therefore $D$ is an implicative filter of $H$.

$(i i) \Rightarrow(i v)$ We have $a \rightarrow 1 \in D$ hence $1 \in H_{a}$. If $x, x \rightarrow y \in H_{a}$ then $a \rightarrow(x \rightarrow y) \in D, a \rightarrow x \in D$ thus $a \rightarrow y \in D$, by (ii), hence $y \in H_{a}$.

$(i v) \Rightarrow(i i)$. Let $x \rightarrow(y \rightarrow z) \in D, x \rightarrow y \in D$. Then $y \rightarrow z \in H_{x}$ and $y \in$ $H_{x}$. Since $H_{x}$ is an implicative filter, we have $z \in H_{x}$ and so $x \rightarrow z \in D$.

The following theorem give a characterization for maximal implicative filter in Hilbert algebras.

Theorem 3.2. [13] Let $D$ be an implicative filter of $H$. Then the following conditions are equivalent:

(i) D is maximal;

(ii) $D$ is proper (i.e. $D \neq H$ ), and if $x, y \notin D$, then $x \rightarrow y \in D$.

The next theorem give another characterization of implicative filters of a bounded Hilbert algebra.

Lemma 3.3. If $D$ is an implicative filter of a bounded Hilbert algebra $H$, then the following conditions are equivalent:

(i) $y \rightarrow x \in D$ implies $((x \rightarrow y) \rightarrow y) \rightarrow x \in D$, for all $x, y \in H$;

(ii) $z \rightarrow(y \rightarrow x) \in D$ and $z \in D$ imply $((x \rightarrow y) \rightarrow y) \rightarrow x \in D$, for all $x, y, z \in H$

(iii) $x^{* *} \rightarrow x \in D$, for every $x \in H$;

(iv) $(x \rightarrow y) \rightarrow x \in D$ implies $x \in D$, for every $x, y \in H$.

Proof. $(i) \Rightarrow(i i)$. Assume that $D$ is an implicative filter and $z \rightarrow(y \rightarrow x) \in$ $D$, and $z \in D$. Then $y \rightarrow x \in D$. Thus $((x \rightarrow y) \rightarrow y) \rightarrow x \in D$, by $(i)$.

$(i i) \Rightarrow($ iii $)$. Suppose $x \in H$. Since $1 \rightarrow(0 \rightarrow x)=1 \in D$, then $x^{* *} \rightarrow x=((x \rightarrow 0) \rightarrow 0) \rightarrow x \in D$. 
(iii) $\Rightarrow($ iv $)$. Let $(x \rightarrow y) \rightarrow x \in D$. We have $(x \rightarrow y) \rightarrow x \leq(x \rightarrow 0) \rightarrow$ $x=x^{*} \rightarrow x$, thus $x^{*} \rightarrow x \in D$. Since $\left(x^{*} \rightarrow x\right) \rightarrow x=x^{* *} \rightarrow x \in D, x^{*} \rightarrow$ $x \in D$ hence $x \in D$.

$(i v) \Rightarrow(i)$. Let $x, y \in H, y \rightarrow x \in D$. Since $x \leq((x \rightarrow y) \rightarrow y) \rightarrow x$, we have $(((x \rightarrow y) \rightarrow y) \rightarrow x) \rightarrow y \leq x \rightarrow y$. Further,

$$
\begin{aligned}
((((x \rightarrow y) \rightarrow y) \rightarrow & x) \rightarrow y) \rightarrow(((x \rightarrow y) \rightarrow y) \rightarrow x) \\
& (x \rightarrow y) \rightarrow(((x \rightarrow y) \rightarrow y) \rightarrow x) \geq \\
& ((x \rightarrow y) \rightarrow y) \rightarrow((x \rightarrow y) \rightarrow x) \geq y \rightarrow x .
\end{aligned}
$$

By the assumption $y \rightarrow x \in D$, thus $((((x \rightarrow y) \rightarrow y) \rightarrow x) \rightarrow y) \rightarrow(((x \rightarrow$ $y) \rightarrow y) \rightarrow x) \in D$. Thus $(((x \rightarrow y) \rightarrow y) \rightarrow x) \in D$.

Therefore, in a bounded Implicative semilattice, we have:

Theorem 3.4. If $D$ is an implicative filter of a bounded Implicative semilattice then the following conditions are equivalent:

(i) $y \rightarrow x \in D$ implies $((x \rightarrow y) \rightarrow y) \rightarrow x \in D$, for all $x, y \in H$;

(ii) $x^{* *} \rightarrow x \in D$, for every $x \in H$;

(iii) If $x \rightarrow u \in D$ and $y \rightarrow u \in D$, then $((x \rightarrow y) \rightarrow y) \rightarrow u \in D$, for every $x, y, u \in H$.

Proof. $(i) \rightarrow($ ii $)$. Let $x \in H$. We have $0 \rightarrow x=1 \in D$. Thus $((x \rightarrow 0) \rightarrow$ $0) \rightarrow x \in D$, for all $x \in H$ by $(i)$. Hence $x^{* *} \rightarrow x \in D$.

$($ ii $) \rightarrow($ iii). Suppose that $x, y, u \in H, x \rightarrow u \in D$ and $y \rightarrow u \in D$. Since $x \rightarrow u \leq u^{*} \rightarrow x^{*}$ and $y \rightarrow u \leq u^{*} \rightarrow y^{*}$, then we have $u^{*} \rightarrow x^{*}, u^{*} \rightarrow y^{*} \in D$. Thus $\left(u^{*} \rightarrow x^{*}\right) \wedge\left(u^{*} \rightarrow y^{*}\right) \in D$. Moreover,

$$
\begin{aligned}
\left(u^{*} \rightarrow x^{*}\right) \wedge\left(u^{*} \rightarrow y^{*}\right) & =u^{*} \rightarrow\left(x^{*} \wedge y^{*}\right) \\
& =u^{*} \rightarrow\left(y^{*} \wedge\left(y^{*} \rightarrow x^{*}\right)\right) \\
& =u^{*} \rightarrow\left(y^{*} \wedge\left(y^{*} \rightarrow(x \rightarrow 0)\right)\right) \\
& =u^{*} \rightarrow\left(y^{*} \wedge\left(x \rightarrow\left(y^{*} \rightarrow 0\right)\right)\right) \\
& =u^{*} \rightarrow\left(y^{*} \wedge\left(x \rightarrow y^{* *}\right)\right) .
\end{aligned}
$$

Further,

$$
\begin{aligned}
\left(u^{*} \rightarrow\left(y^{*} \wedge\left(x \rightarrow y^{* *}\right)\right)\right) \rightarrow\left(u^{*} \rightarrow\left(y^{*} \wedge(x \rightarrow y)\right)\right) & \geq \\
\left(y^{*} \wedge\left(x \rightarrow y^{* *}\right)\right) \rightarrow\left(y^{*} \wedge(x \rightarrow y)\right) & \geq \\
\left(x \rightarrow y^{* *}\right) \rightarrow(x \rightarrow y) & \geq y^{* *} \rightarrow y .
\end{aligned}
$$


Since $y^{* *} \rightarrow y \in D$, thus $u^{*} \rightarrow\left(y^{*} \wedge(x \rightarrow y)\right) \in D$. Also,

$$
\begin{aligned}
u^{*} \rightarrow\left(y^{*} \wedge(x \rightarrow y)\right) & \leq\left(y^{*} \wedge(x \rightarrow y)\right)^{*} \rightarrow u^{* *} \\
& =\left((x \rightarrow y) \rightarrow y^{* *}\right) \rightarrow u^{* *}
\end{aligned}
$$

Thus $\left((x \rightarrow y) \rightarrow y^{* *}\right) \rightarrow u^{* *} \in D$. Further,

$\left(\left((x \rightarrow y) \rightarrow y^{* *}\right) \rightarrow u^{* *}\right) \rightarrow\left(((x \rightarrow y) \rightarrow y) \rightarrow u^{* *}\right) \geq(((x \rightarrow y) \rightarrow y) \rightarrow$ $\left.\left((x \rightarrow y) \rightarrow y^{* *}\right)\right) \geq y \rightarrow y^{* *}=1 \in D$.

Therefore $\left(((x \rightarrow y) \rightarrow y) \rightarrow u^{* *}\right) \in D$. Also, $\left(((x \rightarrow y) \rightarrow y) \rightarrow u^{* *}\right) \rightarrow$ $(((x \rightarrow y) \rightarrow y) \rightarrow u) \geq u^{* *} \rightarrow u \in D$, hence also $(((x \rightarrow y) \rightarrow y) \rightarrow u) \in D$

$($ iii $) \Rightarrow(i)$. In $($ iii $)$ we get $u=x$.

The next theorem we give some equivalent condition for Boolean algebra.

Theorem 3.5. Let $H$ be a bounded Hilbert algebra. Then the following conditions are equivalent:

$(i)((x \rightarrow y) \rightarrow y) \rightarrow x=y \rightarrow x ;$

(ii) $(x \rightarrow y) \rightarrow y=(y \rightarrow x) \rightarrow x ;$

(iii) if $x \rightarrow z \leq y \rightarrow z, z \leq x$, then $y \leq x$;

(iv) if $x \rightarrow z \leq y \rightarrow z, z \leq x, y$, then $y \leq x$;

$(v)$ if $y \leq x$, then $(x \rightarrow y) \rightarrow y \leq x ;$

(vi) $x^{* *}=x$

for all $x, y, z \in H$.

Proof. $(i) \Rightarrow(i i)$.

$$
\begin{aligned}
((x \rightarrow y) \rightarrow y) \rightarrow((y \rightarrow x) \rightarrow x) & =(y \rightarrow x) \rightarrow(((x \rightarrow y) \rightarrow y) \rightarrow x) \\
& =(y \rightarrow x) \rightarrow(y \rightarrow x) \\
& =1 .
\end{aligned}
$$

Thus $((x \rightarrow y) \rightarrow y) \leq((y \rightarrow x) \rightarrow x)$, similarly $((x \rightarrow y) \rightarrow y) \geq((y \rightarrow x) \rightarrow$ $x)$. Therefore $((x \rightarrow y) \rightarrow y)=((y \rightarrow x) \rightarrow x)$.

$(i i) \Rightarrow(i)$. By Proposition 2.2 and $(i)$,

$$
\begin{aligned}
((x \rightarrow y) \rightarrow y) \rightarrow x & =((y \rightarrow x) \rightarrow x) \rightarrow x \\
& =y \rightarrow x
\end{aligned}
$$


$($ ii $) \Rightarrow($ iii $)$. Assume that $x, y \in H$, be such that $x \rightarrow z \leq y \rightarrow z$ and $z \leq x$. By Proposition 2.2

$$
\begin{aligned}
1 & =(x \rightarrow z) \rightarrow(y \rightarrow z) \\
& =y \rightarrow((x \rightarrow z) \rightarrow z) \\
& =y \rightarrow((z \rightarrow x) \rightarrow x) \\
& =y \rightarrow(1 \rightarrow x) \\
& =y \rightarrow x
\end{aligned}
$$

Thus $y \leq x$.

$($ iii $) \Rightarrow($ iv $)$. Is obvious.

$(i v) \Rightarrow(v)$. Assume that $x, y \in H$ and $y \leq x$. By Proposition $2.2(x \rightarrow$ $y) \leq((x \rightarrow y) \rightarrow y) \rightarrow y$, thus $(x \rightarrow y) \rightarrow y \leq x$ by $(i v)$.

$(v) \Rightarrow(v i)$. Since $0 \leq x$ by $(\mathrm{v})(x \rightarrow 0) \rightarrow 0 \leq x$, thus $x^{* *} \leq x$. On the other hand $x \leq x^{* *}$ by Proposition 2.3. Thus $x^{* *}=x$.

$(v i) \Rightarrow(i i)$. See Propositions 2.2 and 2.3.

\section{Positive implicative filters in Hilbert algebras}

In this section, the notion of a positive implicative filter in Hilbert algebras is introduced and its properties are studied.

Definition 4.1. A non- empty subset $F$ of $H$ is called a positive implicative filter if it satisfies:

(i) $1 \in F$,

(ii) $x \rightarrow((y \rightarrow z) \rightarrow y) \in F$ and $x \in F$ imply $y \in F$,

for every $x, y, z \in H$.

Example 4.2. $\quad(i)$ Define

$$
x \rightarrow y= \begin{cases}1 & \text { if } x \leq y \\ y & \text { if } x>y\end{cases}
$$

Then $H=([0,1], \rightarrow, 0,1)$ is a Hilbert algebra. $F=\left[\frac{1}{2}, 1\right]$ is an implicative filter but is not a positive implicative filter, since $\frac{2}{3} \rightarrow\left(\left(\frac{1}{3} \rightarrow \frac{1}{4}\right) \rightarrow\right.$ $\left.\frac{1}{3}\right)=1 \in F$ and $\frac{2}{3} \in F$ but $\frac{1}{3} \notin F$.

(ii) Let $H=\{0, a, b, c, 1\}$, with $0<a, b<c<1$, and a, b are incompatible. With the following operation: 


\begin{tabular}{c|ccccc}
$\rightarrow$ & 0 & $a$ & $b$ & $c$ & 1 \\
\hline 0 & 1 & 1 & 1 & 1 & 1 \\
$a$ & $b$ & 1 & $b$ & 1 & 1 \\
$b$ & $a$ & $a$ & 1 & 1 & 1 \\
$c$ & 0 & $a$ & $b$ & 1 & 1 \\
1 & 0 & $a$ & $b$ & $c$ & 1
\end{tabular}

$H$ is a Hilbert algebra and $\{1, \mathrm{c}\}$ is a positive implicative filter.

(iii) Let $H=\{1, a, b, c\}$. Define $\rightarrow$ by

\begin{tabular}{c|cccc}
$\rightarrow$ & 1 & $a$ & $b$ & $c$ \\
\hline 1 & 1 & $a$ & $b$ & $c$ \\
$a$ & 1 & 1 & $b$ & $c$ \\
$b$ & 1 & $a$ & 1 & $c$ \\
$c$ & 1 & $a$ & $b$ & 1
\end{tabular}

Then $H$ is a Hilbert algebra and by routine calculations we can see that $\{1\}$ is a positive implicative filter.

Proposition 4.3. Any positive implicative filter of $H$ is an implicative filter of $H$.

Proof. Let $x, x \rightarrow y \in F$. Thus $x \rightarrow y=x \rightarrow((y \rightarrow 1) \rightarrow y) \in F$, therefore $y \in F$, since $F$ is a positive implicative filter of $H$.

Remark 4.4. Example 4.2(i) shows that the converse of Proposition 4.3 is not true.

Proposition 4.5. Let $F$ be a positive implicative filter. Then, for all $x, y \in H$,

(i) $(x \rightarrow y) \rightarrow x \in F$, implies $x \in F$.

(ii) $(x \rightarrow y) \rightarrow y \in F$ implies $(y \rightarrow x) \rightarrow x \in F$.

Proof. (i) Let $x, y \in H$ and $(x \rightarrow y) \rightarrow x \in F$. We have $1 \rightarrow((x \rightarrow y) \rightarrow x) \in$ $F$ and $1 \in F$. Thus $x \in F$.

(ii) Let $(x \rightarrow y) \rightarrow y \in F$. From $x \leq(y \rightarrow x) \rightarrow x$ and Proposition 2.2 we conclude that $((y \rightarrow x) \rightarrow x) \rightarrow y \leq x \rightarrow y$. 
Now, consider

$$
\begin{aligned}
(x \rightarrow y) \rightarrow y & \leq(y \rightarrow x) \rightarrow((x \rightarrow y) \rightarrow y) \\
& =(x \rightarrow y) \rightarrow((y \rightarrow x) \rightarrow x) \\
\leq & (((y \rightarrow x) \rightarrow x) \rightarrow y) \rightarrow((y \rightarrow x) \rightarrow x)
\end{aligned}
$$

by Proposition 2.2 and $(*)$. Hence $(((y \rightarrow x) \rightarrow x) \rightarrow y) \rightarrow((y \rightarrow x) \rightarrow x) \in$ $F$. Thus by (i), $(y \rightarrow x) \rightarrow x \in F$, for all $x, y \in H$.

Proposition 4.6. Let $F$ be an implicative filter of $H$ and $(x \rightarrow y) \rightarrow x \in F$, implies $x \in F$, for all $x, y \in H$. Then $F$ is a positive implicative filter of $H$.

Proof. Let $x \in F$ and $x \rightarrow((y \rightarrow z) \rightarrow y) \in F$. Since $F$ is an implicative filter, then $(y \rightarrow z) \rightarrow y \in F$, by hypothesis $y \in F$.

Proposition 4.7. Let $F$ be an implicative filter of $H$ and $(x \rightarrow y) \rightarrow y \in F$ implies $(y \rightarrow x) \rightarrow x \in F$, for all $x, y \in H$. Then $F$ is a positive implicative filter of $H$.

Proof. Let $z \rightarrow((x \rightarrow y) \rightarrow x) \in F$ and $z \in F$. Thus $(x \rightarrow y) \rightarrow x \in F$, since $F$ is an implicative filter. By Proposition 2.2, $(x \rightarrow y) \rightarrow x \leq(x \rightarrow y) \rightarrow$ $((x \rightarrow y) \rightarrow y)$, so $(x \rightarrow y) \rightarrow((x \rightarrow y) \rightarrow y) \in F$, thus $(x \rightarrow y) \rightarrow y \in F$. Therefore

$$
(y \rightarrow x) \rightarrow x \in F
$$

By Proposition $2.2, y \leq x \rightarrow y$, so $(x \rightarrow y) \rightarrow x \leq y \rightarrow x \leq z \rightarrow(y \rightarrow x)$, thus $z \rightarrow(y \rightarrow x) \in F$. Since $z \in F$ and $F$ is an implicative, then $y \rightarrow x \in F$. Hence $x \in F$.

Theorem 4.8. (Extension property for positive implicative filter). Let $F$ be a positive implicative filter. Then every implicative filter $G$ containing $F$ is also a positive implicative filter.

Proof. Let $(y \rightarrow x) \rightarrow x \in G$. It is sufficient to show that $(x \rightarrow y) \rightarrow y \in G$. Let $u=(y \rightarrow x) \rightarrow x$. Thus $u \rightarrow((y \rightarrow x) \rightarrow x)=1 \in F$. So

$$
\begin{aligned}
u \rightarrow((y \rightarrow x) \rightarrow x) & =(u \rightarrow(y \rightarrow x)) \rightarrow(u \rightarrow x) \\
& =(y \rightarrow(u \rightarrow x)) \rightarrow(u \rightarrow x) \in F .
\end{aligned}
$$

So $((u \rightarrow x) \rightarrow y) \rightarrow y \in F$, thus $((u \rightarrow x) \rightarrow y) \rightarrow y \in G$. 
Now, consider

$$
\begin{aligned}
(y \rightarrow x) \rightarrow x & \leq(((y \rightarrow x) \rightarrow x) \rightarrow x) \rightarrow x \\
& \leq(u \rightarrow x) \rightarrow x \\
& \leq(x \rightarrow y) \rightarrow((u \rightarrow x) \rightarrow y) \\
& \leq(((u \rightarrow x) \rightarrow y) \rightarrow y) \rightarrow((x \rightarrow y) \rightarrow y) .
\end{aligned}
$$

So $(((u \rightarrow x) \rightarrow y) \rightarrow y) \rightarrow((x \rightarrow y) \rightarrow y) \in G$. Thus $(x \rightarrow y) \rightarrow y \in G$, since $((u \rightarrow x) \rightarrow y) \rightarrow y \in G$. We get that $(x \rightarrow y) \rightarrow y \in G$.

Theorem 4.9. The following conditions are equivalent:

(i) $\{1\}$ is a positive implicative filter of $H$;

(ii) Every implicative filter of $H$ is a positive implicative filter;

(iii) $H(a)=\{x \in H: x \geq a\}$ is a positive implicative filter, for every $a \in H$;

(iv) $(x \rightarrow y) \rightarrow x=x$, for all $x, y \in H$.

Proof. $(i) \Rightarrow(i i)$. By Theorem 4.8 is clear.

$($ ii $) \Rightarrow($ iii $)$. Let $a \in H$. Since $1 \geq a$, we have $1 \in H(a)$. Let $x, x \rightarrow y \in$ $H(a)$. Then $a \rightarrow x=1, a \rightarrow(x \rightarrow y)=1$ thus $a \rightarrow y=1$, so $a \leq y$. Therefore $y \in H(a)$. Hence $H(a)$ is an implicative filter and by $(i i), H(a)$ is a positive implicative filter.

$($ iii $) \Rightarrow($ iv $)$. We have $(x \rightarrow y) \rightarrow x \in H((x \rightarrow y) \rightarrow x)$. By $($ iii $)$, we have $H((x \rightarrow y) \rightarrow x)$ is a positive implicative filter, then by Proposition $4.5, x \in$ $H((x \rightarrow y) \rightarrow x)$ and so $x \geq(x \rightarrow y) \rightarrow x$. Also we have $x \leq(x \rightarrow y) \rightarrow x$. Hence $x=(x \rightarrow y) \rightarrow x$.

$(i v) \rightarrow(i)$ Let $z \rightarrow((x \rightarrow y) \rightarrow x) \in\{1\}$ and $z=1$. We have $(x \rightarrow y) \rightarrow$ $x \in\{1\}$. By hypothesis $x \in F$.

\section{$5 \quad$ Fantastic filters in Hilbert algebras}

In this section, the notion of fantastic filters in Hilbert algebra is defined. We give relationships between fantastic filters and other types of filters in $H$.

Definition 5.1. A non- empty subset $F$ of $H$ is called a fantastic filter if it satisfies:

(i) $1 \in F$,

(ii) $z \rightarrow(y \rightarrow x) \in F$ and $z \in F$ imply $((x \rightarrow y) \rightarrow y) \rightarrow x \in F$, 
for every $x, y, z \in H$.

Theorem 5.2. Any fantastic filter of $H$ is an implicative filter of $H$.

Proof. Let $x, x \rightarrow y \in F$. Thus $x \rightarrow y=x \rightarrow(1 \rightarrow y) \in F$, therefore $((y \rightarrow 1) \rightarrow 1) \rightarrow y \in F$, hence $y \in F$.

In the following example, we show that the converse of above theorem is not true.

Example 5.3. (i) Let $H=\{1, a, b, c, d\}$. In which $\rightarrow$ is defined by

\begin{tabular}{c|ccccc}
$\rightarrow$ & 1 & $a$ & $b$ & $c$ & $d$ \\
\hline 1 & 1 & $a$ & $b$ & $c$ & $d$ \\
$a$ & 1 & 1 & $b$ & $c$ & $d$ \\
$b$ & 1 & 1 & 1 & $c$ & $d$ \\
$c$ & 1 & 1 & 1 & 1 & $d$ \\
$d$ & 1 & 1 & $b$ & $c$ & 1
\end{tabular}

$(H, \rightarrow, 1)$ is a Hilbert algebra, $F=\{1\}$ is an implicative filter, but is not fantastic filter, since $1 \rightarrow(c \rightarrow b) \in F, 1 \in F$. But $((b \rightarrow c) \rightarrow c) \rightarrow b=$ $b \notin F$.

(ii) Let $H=\{1, a, b, c\}$. The operation $\rightarrow$ defined by:

\begin{tabular}{c|cccc}
$\rightarrow$ & 1 & $a$ & $b$ & $c$ \\
\hline 1 & 1 & $a$ & $b$ & $c$ \\
$a$ & 1 & 1 & $b$ & $c$ \\
$b$ & 1 & $a$ & 1 & $c$ \\
$c$ & 1 & $a$ & $b$ & 1
\end{tabular}

Then $H$ is a Hilbert algebra and $\{1, c\}$ is a fantastic filter.

Now, we give an equivalent condition for fantastic filter.

Theorem 5.4. Let $F$ be an implicative filter of $H$. Then $F$ is fantastic filter if and only if $x \rightarrow y \in F$ implies $((y \rightarrow x) \rightarrow x) \rightarrow y \in F$, for all $x, y \in H$. 
Proof. Let $F$ be a fantastic filter of $H$ and $x \rightarrow y \in F$. Thus $1 \rightarrow(x \rightarrow y)=$ $x \rightarrow y \in F$. Since $1 \in F$, we have $((y \rightarrow x) \rightarrow x) \rightarrow y \in F$.

Conversely, let $F$ be an implicative filter, $z \rightarrow(x \rightarrow y) \in F$ and $z \in F$. Then we have $x \rightarrow y \in F$, thus $((y \rightarrow x) \rightarrow x) \rightarrow y \in F$. Hence $F$ is a fantastic filter.

Theorem 5.5. (Extension property for fantastic filter). Let $F \subseteq G$ where $F$ is a fantastic filter and $G$ is an implicative filter of $H$. Then $G$ is a fantastic filter.

Proof. Let $x, y \in H$ and $z=x \rightarrow y \in G$. We have $x \rightarrow(z \rightarrow y)=z \rightarrow$ $(x \rightarrow y)=1 \in F$. Since $F$ is a fantastic filter, thus $(((z \rightarrow y) \rightarrow x) \rightarrow$ $x) \rightarrow(z \rightarrow y) \in F \subseteq G$. Thus $(((z \rightarrow y) \rightarrow x) \rightarrow x) \rightarrow(z \rightarrow y) \in G$. So $z \rightarrow((((z \rightarrow y) \rightarrow x) \rightarrow x) \rightarrow y) \in G$, by Proposition 2.2. Since $z \in G$, so $((((z \rightarrow y) \rightarrow x) \rightarrow x) \rightarrow y) \in G$. Also since $y \leq z \rightarrow y$, we have $(y \rightarrow x) \rightarrow x \leq((z \rightarrow y) \rightarrow x) \rightarrow x$, thus $(((z \rightarrow y) \rightarrow x) \rightarrow x) \rightarrow y \leq((y \rightarrow$ $x) \rightarrow x) \rightarrow y$. So $((y \rightarrow x) \rightarrow x) \rightarrow y \in G$. Hence $G$ is a fantastic filter.

Corollary 5.6. The implicative filter $\{1\}$ is a fantastic filter of $H$ if and only if every implicative filter of $H$ is a fantastic filter of $H$.

In the following theorem we describe the relationship between positive implicative filters and fantastic filters.

Theorem 5.7. If $F$ is a positive implicative filter of $H$, then $F$ is a fantastic filter of $H$.

Proof. Let $F$ be a positive implicative filter of $H$ and $x \rightarrow y \in F$. We must show that $((y \rightarrow x) \rightarrow x) \rightarrow y \in F$. Since $y \leq((y \rightarrow x) \rightarrow x) \rightarrow y$, we have $(((y \rightarrow x) \rightarrow x) \rightarrow y) \rightarrow x \leq y \rightarrow x$. Further, $(y \rightarrow x) \rightarrow x \leq((((y \rightarrow x) \rightarrow$ $x) \rightarrow y) \rightarrow x) \rightarrow x$ and $x \rightarrow y \leq((y \rightarrow x) \rightarrow x) \rightarrow((y \rightarrow x) \rightarrow y)=(y \rightarrow$ $x) \rightarrow(((y \rightarrow x) \rightarrow x) \rightarrow y) \leq(((y \rightarrow x) \rightarrow x) \rightarrow y) \rightarrow x) \rightarrow(((y \rightarrow x) \rightarrow$ $x) \rightarrow y)$.

By the assumption $x \rightarrow y \in F$, implies

$((((y \rightarrow x) \rightarrow x) \rightarrow y) \rightarrow x) \rightarrow(((y \rightarrow x) \rightarrow x) \rightarrow y) \in F$.therefore $(((y \rightarrow x) \rightarrow x) \rightarrow y) \in F$. Hence $F$ is a fantastic filter.

The converse of Theorem 5.7, is correct in bounded Hilbert algebra (see Proposition 6.13).

Theorem 5.8. $\{1\}$ is a fantastic filter if and only if $((x \rightarrow y) \rightarrow y) \rightarrow x=$ $y \rightarrow x$, for all $x, y \in H$. 
Proof. Let $\{1\}$ be a fantastic filter and $x, y \in H$. We have $x \rightarrow((x \rightarrow$ $y) \rightarrow y)=1 \in\{1\}$, since $x \leq(x \rightarrow y) \rightarrow y$ by Proposition 2.2. Thus $((((x \rightarrow y) \rightarrow y) \rightarrow x) \rightarrow x) \rightarrow((x \rightarrow y) \rightarrow y)=1$. So $((((x \rightarrow y) \rightarrow y) \rightarrow$ $x) \rightarrow x) \leq((x \rightarrow y) \rightarrow y)$. By Proposition 2.2 we have

$((((x \rightarrow y) \rightarrow y) \rightarrow x) \rightarrow x)=((x \rightarrow y) \rightarrow y)$

Also, since $y \leq(x \rightarrow y) \rightarrow y$, we have $((x \rightarrow y) \rightarrow y) \rightarrow x \leq y \rightarrow x$, so $(y \rightarrow x) \rightarrow x \leq(((x \rightarrow y) \rightarrow y) \rightarrow x) \rightarrow x$. Thus $(y \rightarrow x) \rightarrow x \leq(x \rightarrow y) \rightarrow$ $y$, by $(*)$ and similarly $(x \rightarrow y) \rightarrow y \leq(y \rightarrow x) \rightarrow x$. Therefore $(x \rightarrow y) \rightarrow$ $y=(y \rightarrow x) \rightarrow x$. Hence $((x \rightarrow y) \rightarrow y) \rightarrow x=((y \rightarrow x) \rightarrow x) \rightarrow x=y \rightarrow x$, for all $x, y \in H$.

By Theorem 5.4 the converse is clear.

Theorem 5.9. Let $F$ be an implicative filter of $H$. Then $F$ is a fantastic filter if and only if every implicative filter of the quotient algebra $H / F$ is a fantastic filter of $H / F$.

Proof. Assume that $F$ is a fantastic filter of $H$ and $x, y \in H$ be such that $[x] \rightarrow[y]=1$. Then $x \rightarrow y=1$. Thus $((y \rightarrow x) \rightarrow x) \rightarrow y \in F$, then $(([y] \rightarrow[x]) \rightarrow[x]) \rightarrow[y]=[((y \rightarrow x) \rightarrow x) \rightarrow y]=[1]$. Therefore $\{[1]\}$ is a fantastic filter of $H / F$. Thus every implicative filter of $H / F$ is a fantastic filter.

Conversely, consider $x, y \in H$ and $x \rightarrow y \in F$. Then $[x] \rightarrow[y]=[x \rightarrow y]=$ [1]. Thus $(([y] \rightarrow[x]) \rightarrow[x]) \rightarrow[y]=[((y \rightarrow x) \rightarrow x) \rightarrow y]=[1]$, since $\{[1]\}$ is a fantastic filter of $H / F$. Therefore $((y \rightarrow x) \rightarrow x) \rightarrow y \in F$. Hence $F$ is a fantastic filter of $H$.

\section{Boolean filters in Hilbert algebra}

In this section, $H$ is a bounded Hilbert algebra with supremum, unless otherwise is stated.

Definition 6.1. An implicative filter $F$ of $H$ is called a Boolean filter of:

(1) first kind (BF1) if $x^{* *} \rightarrow x \in F$, for all $x \in H$.

(2) second kind (BF2) if $x \vee x^{*} \in F$, for all $x \in H$.

Example 6.2. (i) Let $H=\{0, a, b, c, 1\}$, with $0<a, b<c<1$, but $a, b$ are incompatible. We define 


\begin{tabular}{c|ccccc}
$\rightarrow$ & 0 & $a$ & $b$ & $c$ & 1 \\
\hline 0 & 1 & 1 & 1 & 1 & 1 \\
$a$ & $b$ & 1 & $b$ & 1 & 1 \\
$b$ & $a$ & $a$ & 1 & 1 & 1 \\
$c$ & 0 & $a$ & $b$ & 1 & 1 \\
1 & 0 & $a$ & $b$ & $c$ & 1
\end{tabular}

$(H, \rightarrow, 0,1)$ is a bounded Hilbert algebra with supremum. The implicative filters are $\{1\},\{c, 1\},\{a, c, 1\},\{b, c, 1\}$ and $\{0, a, b, c, 1\}$. The implicative filters $\{c, 1\},\{a, c, 1\},\{b, c, 1\},\{0, a, b, c, 1\}$ are $B F 1$ and $B F 2,\{1\}$ is a $B F 1$ but is not a $B F 2$.

(ii) Let $H=\{0, a, b, 1\}$. $\rightarrow$ defined by:

\begin{tabular}{c|cccc}
$\rightarrow$ & 0 & $a$ & $b$ & 1 \\
\hline 0 & 1 & 1 & 1 & 1 \\
$a$ & $b$ & 1 & $b$ & 1 \\
$b$ & $a$ & $a$ & 1 & 1 \\
1 & 0 & $a$ & $b$ & 1
\end{tabular}

Then $H$ is a bounded Hilbert algebra with supremum, $F=\{1\}$ is a $B F 1$, but is not a $B F 2$, because $a \vee b=1$ but $a, b \notin F$. The filters $\{a, 1\},\{b, 1\},\{0, a, b, 1\}$ are $B F 1$ and $B F 2$, but their intersection $\{1\}$ is not a $B F 2$.

(iii) Let $H=\{0, a, 1\}$, where $0<a<1$. The operation $\rightarrow$ defined by:

\begin{tabular}{c|ccc}
$\rightarrow$ & 0 & $a$ & 1 \\
\hline 0 & 1 & 1 & 1 \\
$a$ & 0 & 1 & 1 \\
1 & 0 & $a$ & 1
\end{tabular}

Notice that $\{1\}$ is not $B F 1$ and $B F 2$, because $a^{* *} \rightarrow a=a \notin\{1\}$ and $a \vee a^{*}=a \notin\{1\}$.

(iv) Let $H=\{0, a, b, c, 1\}$. We define 


\begin{tabular}{c|ccccc}
$\rightarrow$ & 0 & $a$ & $b$ & $c$ & 1 \\
\hline 0 & 1 & 1 & 1 & 1 & 1 \\
$a$ & 0 & 1 & $b$ & $b$ & 1 \\
$b$ & 0 & $a$ & 1 & $a$ & 1 \\
$c$ & 0 & 1 & 1 & 1 & 1 \\
1 & 0 & $a$ & $b$ & $c$ & 1
\end{tabular}

$(H, \rightarrow, 0,1)$ is a bounded Hilbert algebra with supremum. The implicative filter $\{1\}$ is not none of $B F 1$ and $B F 2$.

It is easy to see that a Boolean filter of the second kind is a Boolean filter of the first kind.

Proposition 6.3. Every Boolean filter of the second kind of $H$ is also a Boolean filter of the first kind.

Proof. Since $x \vee x^{*} \leq\left(x^{*} \rightarrow x\right) \rightarrow x=x^{* *} \rightarrow x$, for all $x \in H$. Thus $x^{* *} \rightarrow x \in F$, for all $x \in H$. Hence $F$ is a Boolean filter of the first kind.

In the following example, we show that the converse of above proposition is not true.

Example 6.4. Let $H=\{0, a, b, c, 1\}$. Define

\begin{tabular}{c|ccccc}
$\rightarrow$ & 0 & $a$ & $b$ & $c$ & 1 \\
\hline 0 & 1 & 1 & 1 & 1 & 1 \\
$a$ & $a$ & 1 & 1 & 1 & 1 \\
$b$ & $a$ & $c$ & 1 & $c$ & 1 \\
$c$ & $a$ & $b$ & $b$ & 1 & 1 \\
1 & 0 & $a$ & $b$ & $c$ & 1
\end{tabular}

$H$ is a bounded Hilbert algebra with supremum, since $x^{* *} \rightarrow x=1$ for all $x \in H$, thus $\{1\}$ is a Boolean filter of the first kind of $H$, but $a \vee a^{*}=a \vee a=$ $a \notin\{1\}$.

A Tarski algebra [7] is a Hilbert algebra $(H, \rightarrow, 1)$ such that $(a \rightarrow b) \rightarrow$ $a=(b \rightarrow a) \rightarrow a$ for all $a, b, c \in H$. It is known that $H$ is a join-semilattice under the operation $\vee$ which defined by $a \vee b=(a \rightarrow b) \rightarrow a$. Thus, $H$ is a Hilbert algebra with supremum.

Note. If $H$ is a Tarski algebra, the converse of Proposition 6.3 is true, since $x \vee x^{*}=\left(x \rightarrow x^{*}\right) \rightarrow x=\left(x^{*} \rightarrow x\right) \rightarrow x=x^{* *} \rightarrow x$. 
Proposition 6.5. (Extension property for Boolean filter)

(i) If $F$ is a Boolean filter of the first kind of $H$, then every implicative filter $G$ of $H$ containing $F$ is also a Boolean filter of the first kind.

(ii) If $F$ is a Boolean filter of the second kind, then every implicative filter $G$ containing $F$ is also a Boolean filter of the second kind.

Proof. (i) Let $F$ be a Boolean filter of the first kind and $F \subseteq G$. We have $x^{* *} \rightarrow x \in F$, for all $x \in H$. Thus $x^{* *} \rightarrow x \in G$, for all $x \in H$. Therefore $G$ is a Boolean filter of the first kind.

(ii) Let $F$ be a Boolean filter of the second kind and $F \subseteq G$. We have $x^{*} \vee x \in F$, for all $x \in H$. Thus $x^{*} \vee x \in G$, for all $x \in H$. Therefore $G$ is a Boolean filter of the second kind.

Theorem 6.6. Let $F \subseteq H$ be an implicative filter. Then

(i) $F$ is a Boolean filter of the first kind if and only if $H / F$ is a Boolean algebra.

(ii) if $F$ is a Boolean filter of the second kind, then $H / F$ is a Boolean algebra.

Proof. Let $x \in H$. By Theorem 2.14,

(i)

$$
\begin{aligned}
H / F \text { is a Boolean algebra } & \Longleftrightarrow[x]^{* *}=[x] \\
& \Longleftrightarrow\left[x^{* *}\right]=[x] \\
& \Longleftrightarrow x^{* *} \rightarrow x \in F, x \rightarrow x^{* *} \in F \\
& \Longleftrightarrow x^{* *} \rightarrow x \in F, 1 \in F \\
& \Longleftrightarrow x^{* *} \rightarrow x \in F \\
& \Longleftrightarrow F \text { is a Boolean filter of the first kind. }
\end{aligned}
$$

(ii) By Proposition 6.3 and $(i)$ is clear.

In the following example, we show that the converse of Theorem 6.6(ii) is not true.

Example 6.7. In Example 6.2(ii), $F=\{1\}$ is not a Boolean filter of the second kind of $H$, but $H / F$ is a Boolean algebra. 
Proposition 6.8. In a bounded Hilbert algebra $H$, positive implicative filter and Boolean filter of the first kind are equivalent.

Proof. Let $F$ be a positive implicative filter. We have $\left(x \rightarrow x^{*}\right) \rightarrow x^{*}=1 \in F$, because $\left(x \rightarrow x^{*}\right) \rightarrow x^{*}=x^{*} \rightarrow x^{*}=1$. By Proposition 4.5, we have $\left(x^{*} \rightarrow x\right) \rightarrow x \in F$, for all $x \in H$. Thus $x^{* *} \rightarrow x \in F$, for all $x \in H$. Therefore $F$ is a Boolean filter of the first kind.

Conversely, let $(x \rightarrow y) \rightarrow x \in F$. We have $(x \rightarrow y) \rightarrow x \leq(x \rightarrow 0) \rightarrow x=$ $x^{*} \rightarrow x$, hence $x^{*} \rightarrow x \in F$. Since $\left(x^{*} \rightarrow x\right) \rightarrow x=x^{* *} \rightarrow x \in F, x^{*} \rightarrow x \in F$ thus $x \in F$. Therefore $F$ is a positive implicative filter.

Proposition 6.9. In a bounded Hilbert algebra with supremum $H$ every Boolean filter of the second kind is a positive implicative filter.

In the following example, we show that the converse of Proposition 6.9 is not true.

Example 6.10. In Example 6.2, $(i),\{1\}$ is a positive implicative filter, but is not a Boolean filter of the second kind.

Proposition 6.11. If $F$ is a fantastic filter in a bounded Hilbert algebra $H$, then $F$ is a Boolean filter of the first kind.

Proof. Let $F$ be a fantastic filter in a bounded Hilbert algebra $H$. By Theorem 5.4, $x \rightarrow y \in F$ implies $((y \rightarrow x) \rightarrow x) \rightarrow y \in F$. For $x=0$ we obtain $y^{* *} \rightarrow y \in F$, for all $y \in H$, then $F$ is a Boolean filter of the first kind.

Example 6.12. In Example 5.3(ii), $\{c, 1\}$ is a fantastic filter but is not a Boolean filter of the first kind since $H$ is not bounded.

Proposition 6.13. In a bounded Hilbert algebra $H$, positive implicative filter and fantastic filter are equivalent.

Open problem. Does the Proposition 6.13 hold in every Hilbert algebra?

Theorem 6.14. In a bounded Hilbert algebra $H$, positive implicative filter, fantastic filter and Boolean filter of the first kind are equivalent.

Theorem 6.15. Let $H$ be a bounded Hilbert algebra. The following conditions are equivalent:

(i) $\{1\}$ is a Boolean filter of the first kind,

(ii) Every implicative filter of $H$ is a Boolean filter of the first kind,

(iii) $H(a)=\{x \in H: x \geq a\}$ is a Boolean filter of the first kind, for every $a \in H$, 
(iv) $(x \rightarrow y) \rightarrow x=x$ for all $x, y \in H$,

(v) $x^{* *}=x$,

(vi) $H$ is a Boolean algebra.

Theorem 6.16. Let $H$ be a bounded Hilbert algebra with supremum. The following conditions are equivalent:

(i) $\{1\}$ is a Boolean filter of the second kind,

(ii) Every implicative filter of $H$ is a Boolean filter of the second kind,

(iii) $H(a)=\{x \in H: x \geq a\}$ is a Boolean filter of the second kind, for every $a \in H$.

Proof. $(i) \Rightarrow(i i)$. By Proposition 6.5.

(ii) $\Rightarrow($ iii $)$. Let $a \in H$. Since $1 \geq a$, we have $1 \in H(a)$. Let $x, x \rightarrow y \in$ $H(a)$, then $a \rightarrow x=1, a \rightarrow(x \rightarrow y)=1$ thus $a \rightarrow y=1$, so $a \leq y$. Therefore $y \in H(a)$. Hence $H(a)$ is an implicative filter and by $(i i), H(a)$ is a Boolean filter of the second kind.

$($ iii $) \Rightarrow($ i $) .\{1\}=\{x \in H: x \geq 1\}=H(1)$.

Theorem 6.17. Let $H$ be a bounded Hilbert algebra. Then $\{1\}$ is a Boolean filter of the first kind of $H$ if and only if $((x \rightarrow y) \rightarrow y) \rightarrow x=y \rightarrow x$, for all $x, y \in H$.

Proof.

$\{1\}$ is a Boolean filter of the first kind $\Longleftrightarrow\left(x^{* *} \rightarrow x\right) \in\{1\}$

$$
\begin{aligned}
& \Longleftrightarrow \quad x^{* *} \leq x \\
& \Longleftrightarrow \quad x^{* *}=x \\
& \Longleftrightarrow \quad((x \rightarrow y) \rightarrow y) \rightarrow x=y \rightarrow x
\end{aligned}
$$

for all $x, y \in H$.

Theorem 6.18. Let $F$ be a maximal filter of a bounded Hilbert algebra $H$. Then $F$ is a Boolean filter of the first kind.

Proof. Let $x \notin F$. By Theorem 2.19 we get that $x^{*} \in F$. So $x^{*} \leq\left(x^{*} \rightarrow x\right) \rightarrow$ $x$, thus $x^{* *} \rightarrow x \in F$. Hence $F$ is a Boolean filter of the first kind.

Theorem 6.19. Let $F$ be a maximal filter of a bounded Hilbert algebra with supremum $H$. Then $F$ is a Boolean filter of the second kind. 
Proof. Let $x \notin F$. By Theorem 2.19 we get that $x^{*} \in F$, so $x^{*} \leq x \vee x^{*}$, thus $x \vee x^{*} \in F$. Therefore $F$ is a Boolean filter of the second kind.

Corollary 6.20. If $F$ is a maximal filter, then $H / F$ is a Boolean algebra.

Proof. Let $F$ be a maximal filter. By Theorem $6.18, F$ is Boolean filter of the first kind and by Theorem 6.6(i), $H / F$ is a Boolean algebra.

\section{Prime filters in Hilbert algebra}

In this section $H$ is a Hilbert algebra with supremum, unless otherwise is stated.

Definition 7.1. An implicative filter $F$ of $H$ is called a prime filter of:

(1) first kind (PF1), if $x \vee y \in F$ implies $x \in F$ or $y \in F$, for all $x, y \in H$.

(2) second kind (PF2), if $x \rightarrow y \in F$ or $y \rightarrow x \in F$, for all $x, y \in H$.

(3) third kind $(P F 3)$, if $(x \rightarrow y) \vee(y \rightarrow x) \in F$, for all $x, y \in H$.

Example 7.2. (i) In Example $6.2(i)$, the implicative filters are $\{1\}$, $\{c, 1\},\{a, c, 1\},\{b, c, 1\}$ and $\{o, a, b, c, 1\}$. Remark that $\{1\}$ is $P F 1$, but is not a $P F 2$, since $a \rightarrow b=b \notin\{1\}, b \rightarrow a=a \notin\{1\}$. Also note that $\{1\}$ is not $P F 3$, since $(a \rightarrow b) \vee(b \rightarrow a)=b \vee a=c$. The implicative filter $\{c, 1\}$ is $P F 3$, but is not $P F 2$ and $P F 1$, since $a \rightarrow b$ and $b \rightarrow a \notin\{c, 1\}$ also, $a \vee b=c \in\{c, 1\}$ but $a, b \notin\{c, 1\}$. The implicative filters $\{a, c, 1\},\{b, c, 1\},\{o, a, b, c, 1\}$ are $P F 1, P F 2, P F 3$, but their intersection $\{c, 1\}$ are not $P F 2, P F 1$.

(ii) In Example $6.2(i i), F=\{1\}$ is a Boolean filter of the first kind, but is not $P F 1$, because $a \vee b=1$ but $a, b \notin F$. The filters $\{a, 1\},\{b, 1\},\{o, a, b, 1\}$ are $P F 1, P F 2, P F 3, B F 1$ and $B F 2$, but their intersection $\{1\}$ is not $P F 1, P F 2$ and $B F 2$.

(iii) In Example 6.2 (iii), $F=\{1\}$ is a prime filter of the first kind, but is not $B F 1$, because $a \vee a^{*}=a \notin\{1\}$.

(iv) In Example $6.2(i v),(H, \rightarrow, 0,1)$ is a bounded Hilbert algebra with supremum. The implicative filter $\{1\}$ is $P F 3$, but is not $P F 1, P F 2, B F 1$ and $B F 2$.

In the following theorem we state the relationship between all kinds of prime filters. 
Theorem 7.3. For an implicative filter $P$ of $H$, consider the following assertions:

(1) $P$ is a prime filter of the first kind;

(2) $P$ is a prime filter of the second kind;

(3) $P$ is a prime filter of the third kind.

Then

(i) $(2) \Rightarrow(1)$ but $(1) \nRightarrow(2)$;

(ii) $(2) \Rightarrow(3)$ but $(3) \nRightarrow(2)$;

(iii) $(1)+(3) \Leftrightarrow(2)$.

Proof. (i). (2) $\Rightarrow(1)$ Suppose $F$ is a prime filter of the second kind of $H$ and $x \vee y \in F$. We know that $F$ contains $x \rightarrow y$ or $y \rightarrow x$. Without loss of generality, we assume that $x \rightarrow y \in F$. Since $x \vee y \leq(x \rightarrow y) \rightarrow y \in F$, therefore $y \in F$. Thus $F$ is a prime filter of the first kind.

$(1) \nRightarrow(2)$ By Example 7.2(i), $H=\{0, a, b, c, 1\}$, is a Hilbert algebra, $\{1\}$ is a prime filter of the first kind, but $b=a \rightarrow b \notin\{1\}, a=b \rightarrow a \notin\{1\}$

(ii). (2) $\Rightarrow(3)$ Suppose that $F$ is a prime filter of the second kind of $H$. We know that $F$ contains $x \rightarrow y$ or $y \rightarrow x$. Since $x \rightarrow y, y \rightarrow x \leq(x \rightarrow$ $y) \vee(y \rightarrow x) \in F$. Thus $F$ is a prime filter of the first kind.

$(3) \nRightarrow(2)$ Consider $H$ in Example 7.2(i). Then $F=\{1, c\}$ is a prime filter of the third kind, but $a \rightarrow b$ and $b \rightarrow a \notin\{c, 1\}$.

(iii). Suppose that $F$ is a prime filter of the third kind of $H$. We know that $(x \rightarrow y) \vee(y \rightarrow x) \in F$, for all $x, y \in H$. Now, we have $x \rightarrow y \in F$ or $y \rightarrow x \in F$, since $F$ is a prime filter of the first kind of $H$. Therefore $F$ is a prime filter of the second kind of $H$.

Converse is clear.

Proposition 7.4. If $H$ is prelinear, then every prime filter of the first kind is a prime filter of the second kind.

Proof. Now, suppose that $F$ is a prime filter of the first kind of $H$ and $x, y \in H$. Since $(x \rightarrow y) \vee(y \rightarrow x)=1$, either $x \rightarrow y$ or $y \rightarrow x$ must be in $F$.

Remark 7.5. Let $F$ be a prime filter of the first kind of a bounded Hilbert algebra with supremum $H$. If $(x \rightarrow y) \vee(y \rightarrow x)=1$, then $x \rightarrow y \in F$ or $y \rightarrow x \in F$, for all $x, y \in H$. 
Proposition 7.6. (Extension property for prime filter).

(i) If $F$ is a prime filter of the second kind, then every implicative filter $G$ containing $F$ is also a prime filter of the second kind.

(ii) If $F$ is a prime filter of the third kind, then every implicative filter $G$ containing $F$ is also a prime filter of the third kind.

Example 7.7. In Example 7.2(i), $\{1\}$ is $\mathrm{PF} 1$, but $\{c, 1\}$ is not PF1. So the extension property does not hold for PF1.

Theorem 7.8. Every maximal filter of $H$ is a prime filter of the second kind.

Proof. Assume $F$ is a maximal filter of $H$. By Theorem 3.2, for $x, y \in H$, we have $x \rightarrow y \in F$ or $y \rightarrow x \in F$. Therefore $F$ is a prime filter of the second kind.

Corollary 7.9. Every maximal filter of $H$ is a prime filter of the first kind.

In the following example, we show that the converse of Theorem 6.18 and Corollary 7.9 is not true. Also we show that extension property for prime filters is not hold.

Example 7.10. In Example 7.2(i), $H=\{0, a, b, c, 1\}$ is a Hilbert algebra, $\{1\}$ is a prime filter of the first kind. Since $c^{* *} \rightarrow c=1 \rightarrow c=c \notin\{1\}$, so $\{1\}$ isn't a Boolean filter of the first kind, thus is not a maximal filter. Also $\{c, 1\}$ is Boolean filter of the first kind but it is not a prime filter of the first kind since $a \vee b=c \in\{c, 1\}$ but $a, b \notin\{c, 1\}$. Also $\{c, 1\}$ is not a maximal filter, since $a \rightarrow b=b \notin\{c, 1\}$.

Theorem 7.11. Let $(H \rightarrow, 1)$ be a Tarski algebra where $H$ is bounded. An implicative filter $F$ is maximal filter if and only if $F$ is prime and Boolean filter of the first kind of $H$.

Proof. For $x \in H$, we have $x \vee x^{*}=\left(x \rightarrow x^{*}\right) \rightarrow x=\left(x^{*} \rightarrow x\right) \rightarrow x=x^{* *} \rightarrow$ $x \in F$, since $F$ is a Boolean filter of the first kind. Thus $x \in F$ or $x^{*} \in F$, because $F$ is a prime filter of the first kind of $H$. Therefore, $F$ is a maximal filter of $H$.

The converse is clear.

Theorem 7.12. Let $F$ be an implicative filter of bounded Hilbert algebra $H$. $F$ is maximal filter if and only if $F$ is a prime filter of the first kind and a Boolean filter of the second kind. 
Proof. For $x \in H$, we have $x \vee x^{*} \in F$, since $F$ is a Boolean filter of the second kind. Thus $x \in F$ or $x^{*} \in F$, because $F$ is a prime filter of the first kind of $H$. Therefore, $F$ is a maximal filter of $H$.

The converse is clear.

Theorem 7.13. Every Boolean filter of the second kind is a prime filter of the third kind.

Proof. For any two elements $x$ and $y$ we have $x \leq y \rightarrow x$ and $x^{*} \leq x \rightarrow y$, it follows that every Boolean filter of the second kind of a Hilbert algebra is also a prime filter of the third kind.

Theorem 7.14. For a proper implicative filter $P$ of $H$ consider the following assertions:

(1) $P$ is a prime filter of the first kind;

(2) If $a, b \in H$, and $a \vee b=1$, then $a \in P$ or $b \in P$;

(3) For all $a, b \in H, a \rightarrow b \in P$ or $b \rightarrow a \in P$;

(4) $H / P$ is a chain.

Then

(i) $(1) \Rightarrow(2)$ but $(2) \nRightarrow(1),(2) \nRightarrow(3),(2) \nRightarrow(4)$;

(ii) (3) $\Rightarrow(1)$ but $(1) \nRightarrow(3)$;

(iii) $(4) \Rightarrow(1)$ but $(1) \nRightarrow(4)$;

Proof. $(i) .(1) \Rightarrow(2)$ is clear, since $1 \in P$.

Consider $H$ in Example $7.2(i)$. Then

(a) $(2) \nRightarrow(1)$ The implicative filter $F=\{1, c\}$ is not prime filter of the first kind, but if $a \vee b=1$, then $a=1$ or $b=1$, hence $a \in F$ or $b \in F$.

(b) For implicative filter $F=\{1\}$, we have, if $a \vee b=1$, then $a=1$ or $b=1$, hence $a \in F$ or $b \in F$. But,

(b1) $(2) \nRightarrow(3)$ Since $a \rightarrow b=b \notin F$ and $b \rightarrow a=a \notin F$.

(b2) $(2) \nRightarrow(4)$ Sinse $H / F \cong H$ is not a chain. 
(ii). $(3) \Rightarrow(1)$. See Theorem $7.3(i)$.

$(1) \nRightarrow(3)$. In Example $7.2(i),\{1\}$ is a prime filter of the first kind, but $a \rightarrow b \notin\{1\}, b \rightarrow a \notin\{1\}$.

(iii). Let $a, b \in H$. Since $H / P$ is supposed chain, $a / P \leq b / P$ or $b / P \leq a / P$ $\Leftrightarrow a \rightarrow b \in P$ or $b \rightarrow a \in P$ and we apply Theorem7.3(ii).

$(1) \nRightarrow(4)$. In Example $7.2(i), P=\{1\}$ is prime filter of the first kind, but $H / P \approx H$ is not a chain.

Remark 7.15. In Theorem 7.14, if $(a \rightarrow b) \vee(b \rightarrow a)=1$, then $(1) \Leftrightarrow(3) \Leftrightarrow$ (4).

Theorem 7.16. Let $P$ be a proper implicative filter of $H$. Then $P$ is a prime filter of the second kind if and only if $H / P$ is a chain.

Proof.

$P$ is a prime filter of the second kind $\Longleftrightarrow a \rightarrow b \in P$ or $b \rightarrow a \in P$

$\Longleftrightarrow a / P \leq b / P$ or $b / P \leq a / P$

$\Longleftrightarrow H / P$ is a chain.

By summarizing all results, we see that the following different situations can occur for a filter $F$ of a bounded $H$ :

- $F$ is a implicative filter of $H$, but $F$ is not $P F 1, P F 2, P F 3, B F 1$ nor $B F 2$;

- $F$ is $P F 1$ but not $P F 2, P F 3, B F 1$ nor $B F 2$;

- $F$ is $P F 3$ but not $P F 1, P F 2, B F 1$ nor $B F 2$;

- $F$ is $P F 2$ (and therefore also $P F 2$ and $P F 3$ ), but not $B F 1$ nor $B F 2$;

- $F$ is $B F 2$ and $P F 3$, but not $P F 1, P F 2$ nor $B F 2$;

- $F$ is $B F 1, B F 2, P F 1, P F 2$, and $P F 3$.

Figure 1 gives a schematic summary of these situations. If $A \leq B$ in this lattice, this means " every filter which is $B$ is also $A$ ". Also $A \vee B=C$ in this lattice, this means "every filter which is $A$ as well as $B$, also $C$ ".

For every implicative filter $F$ of $H$, we have: 


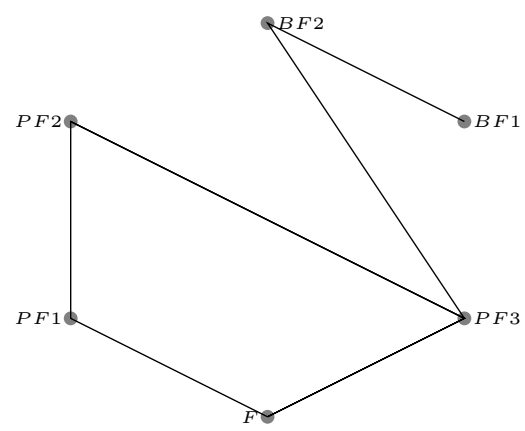

Figure 1:

- The quotient algebra $H / F$ is linear if and only if $F$ is a prime filter of the second kind of $H$.

- $[1]_{F}$ is $\vee_{F^{-}}$irreducible in the quotient algebra $H / F$ if and only if is a prime filter of the first kind of $H$.

- The quotient algebra $H / F$ is prelinear if and only if $F$ is a prime filter of the third kind of $H$.

- The quotient algebra $H / F$ is a Boolean algebra if and only if $F$ is a Boolean filter of the first kind of $H$.

- The quotient algebra $H / F$ is a Boolean algebra if $F$ is a Boolean filter of the second kind of $H$.

\section{Conclusion and future research}

In this paper, we introduced the notions of positive implicative filters, fantastic filters, Boolean filters and prime filters in Hilbert algebra. We proved some theorems which determines the relationship between these filters and other types of filters in a Hilbert algebra and by some examples we showed that these notions are different.

In the following diagram, we summarize the results of this paper and the previous results in this field and we give the relationships between implicative filters, positive implicative filters, fantastic filters, maximal filters, prime filters and Boolean filters. In this diagram, we show that "positive implicative filter" with "positive filter", "Boolean filter of the first kind" with "Boolean filter(1)", "Boolean filter of the second kind" with "Boolean filter(2)", "prime filter of the first kind" with "Prime filter(1)", "prime filter of the second kind" with "Prime 
filter(2)" and "prime filter of the third kind" with "Prime filter(3)". The mark $A \longrightarrow B(A \stackrel{a}{\longrightarrow} B$ or $A \underset{a}{\longrightarrow} B)$, means that $A$ implies $B$ (respectively, $A$ implies $B$ with the condition " $a$ ").

The results of this paper will be devoted to study of Hilbert algebra, intuitionistic's logic which are different extensions of basic logic.

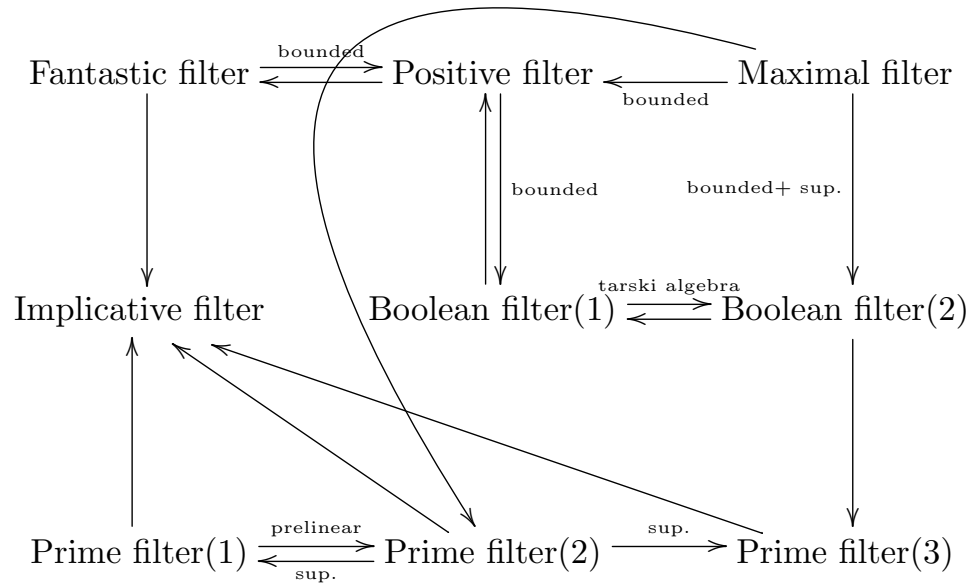

Acknowledgement. The authors would like to express their gratitude to the Editor in Chief Prof. Cristina Flaut and anonymous referees for their comments and suggestions which improved the paper.

\section{References}

[1] Buşneag D., Categories of algebraic logic, Ed. Academiei Române, 2006.

[2] Buşneag D., Contributions to the study of Hilbert algebras (in romanian), PhD Thesis, Univ. of Bucharest, 1985. 
[3] Buşneag D., On the maximal deductive system of a bounded Hilbert algebra, Bull. Math. Soc. Sci. Math. Roumanie, Tome 31(79), nr. 1(1987), 9-21.

[4] Buşneag D., Ghita M., Some latticial properties of Hilbert algebras, Bull. Math. Soc. Sci. Math. Roumanie Tome 53(101) No. 2(2010), 87-107.

[5] Celani S. A., $\alpha$-Ideal and $\alpha$ - Deductive Systems in Bounded Hilbert algebras, J. of Mult. valued Logic and Soft Computing, Vol. o, (2013), $1-18$.

[6] Celani S. A., Jansana R., On the free implicative semilattice extension of a Hilbert algebra, Mathematical Logic Quarterly 58(3), (2012), 1-20.

[7] Celani S. A., Montangie D., Hilbert algebras with supremum, Algebra Universalis, 67 (2012), 237-255.

[8] Diego A., Sur les algebres de Hilbert, Collection de Logique Mathematique, Edition Hermann, Serie A, XXI, 1966.

[9] Figallo A. V., Ramón G. Z., Saad S., A note on the Hilbert algebras with infimum, Math. Contemp. 24(2003), 23-37.

[10] Gluschankof D., Tilli M., Maximal deductive systems and injective objects in the category of Hilbert algebras, Zeitschrift für mathematische Logik und Grundlagen der Mathematik 34, No.3 (1988), 213-220.

[11] Hong S. M., Jun Y. B., On deductive systems of Hilbert algebras, Comm. Korean Math. Soc. 11:3(1996), 595-600.

[12] Köhler P., Brouwerian semilattices. Trans. Amer. Math. Soc. Vol.268,(1981), 103-126.

[13] Monteiro A., Sur les algèbres de Heyting symétriques. Portugaliae Mathematica 39 (1980), fasc. 1-4.

[14] Nemitz W.C., Implicative semi-lattices. Trans. Amer. Math. Soc. $117,(1965), 128-142$.

[15] Piciu D., Algebras of fuzzy logic, Ed. Universitaria, Craiova (2007).

[16] Porta H., Sur quelques algèbres de la Logique.Portugaliae Mathematica. Vol 40, Fasc 1(1981) 41-77.

[17] Van Gasse B., Deschrijver G., Cornelis C., Kerre E.E., Filters of residuated lattices and triangle algebra, Inf. Sci. 180(16) (2010) 3006-3020. 
Ali Soleimani Nasab*,

Department of Pure Mathematics,

Faculty of Mathematics and Computer, Shahid Bahonar University of Ker-

man,

Kerman, Iran.

Email: asoleimaninasab@math.uk.ac.ir

* Shahid Chamran Faculty, Technical and Vocational University, Kerman, Iran e-mail: asoleimaninasab@gmail.com(permanent address)

Arsham Borumand Saeid,

Department of Pure Mathematics,

Faculty of Mathematics and Computer, Shahid Bahonar University of Kerman,

Kerman, Iran.

Email: arsham@mail.uk.ac.ir 